\title{
Predictors of Permanent Pacemaker Insertion after TAVR: A Systematic Review and Updated Meta-Analysis
}

\author{
SUGANDHI MAHAJAN ${ }^{1}$, Rahul Gupta ${ }^{2}$, Aaqib Malik ${ }^{3}$, Pranav Mahajan ${ }^{1}$, Surya \\ Aedma $^{1}$, Wilbert Aronow ${ }^{4}$, Sanjay Mehta ${ }^{1}$, and Dhanunjaya Lakkireddy ${ }^{5}$ \\ ${ }^{1}$ Carle Foundation Hospital \\ ${ }^{2}$ Lehigh Valley Health Network \\ ${ }^{3}$ Westchester Medical Center Health Network \\ ${ }^{4}$ Westchester Medical Center \\ ${ }^{5}$ The Kansas City Heart Rhythm Institute (KCHRI) @ HCA MidWest
}

September 10, 2020

\begin{abstract}
Objectives: The aim of this analysis was to evaluate the predictors associated with increased risk of permanent pacemaker implantation (PPMI) following Transcatheter Aortic Valve Replacement (TAVR). Background: While TAVR has evolved as the standard of care for patients with severe aortic stenosis, conduction abnormalities leading to the need for PPMI is one of the most common post-procedural complications. Methods: A systematic literature search was performed to identify relevant trials from inception to May 2020. Summary effects were calculated using a DerSimonian and Laird random-effects model as odds ratio with $95 \%$ confidence intervals for all the clinical endpoints. Results: 37 observational studies with 71,455 patients were identified. The incidence of PPMI following TAVR was $22 \%$. Risk was greater in men and increased with age. Patients with diabetes mellitus, presence of right bundle branch block, baseline atrioventricular conduction block, and left anterior fascicular block were noted to be at higher risk. Other significant predictors include the presence of high calcium volume in the area below the left coronary cusp and non-coronary cusp, use of self-expandable valve over balloon-expandable valve, depth of implant, valve size/annulus size, pre-dilatation balloon valvuloplasty and post-implant balloon dilation. Conclusion: Fourteen factors were found to be associated with increased risk of PPMI after TAVR, suggesting early identification of high-risk populations and targeting modifiable risk factors may aid in reducing the need for this post TAVR PPMI.
\end{abstract}

\section{Introduction}

Over the past decade, Transcatheter Aortic Valve Replacement (TAVR) has evolved as the standard of care for patients with severe aortic stenosis (AS). With the expansion of its use to patients with low surgical risk the volume of TAVR procedures has increased, as has the rate of associated complications (1). Conduction abnormalities leading to permanent pacemaker implantation (PPMI) is one of the most common (2). These abnormalities are believed to occur due to ischemia, hemorrhage, edema, or trauma either during or after the procedure (3). Even with the advances in both technology and procedural technique, and with the use of newer generation valves, the rate of PPMI following TAVR remains high. However, the rate differs between the two most widely used bioprosthetic valves; the balloon-expandable valve (BEV) and the selfexpandable valve (SEV) (4). The clinical impact of PPMI is of significant concern. This is not only due to its high incidence but also to the potentially adverse effects on outcomes and the side effects of long-term right ventricular pacing (5). The aim of our meta-analysis was to systematically review and evaluate the predictors associated with increased risk.

\section{Methods}




\section{Study Selection and Data Abstraction}

PubMed and Embase were searched from the inception through May 2020. The keywords of transcatheter aortic valve replacement OR transcatheter aortic valve implantation OR permanent pacemaker OR atrioventricular conduction OR cardiac conduction disturbance were used to identify relevant studies. References from the retrieved articles were examined for further identification of potentially relevant studies. The search methodology was not limited to a specific population or clinical presentation. After deduplication, the study titles and abstracts were screened for relevance. Full manuscripts of potentially relevant studies were then reviewed by two independent authors (SM and RG). Any discrepancies were resolved through consensus and arbitration by a third investigator (AM). Studies were considered for inclusion if they consisted of patients who underwent PPMI for any indication within 30 days after TAVR. We included studies that listed quantitative raw data which enabled the calculation of odds ratios (OR) as predictors. Only studies that listed multivariate analysis of the predictors of interest were included; those with only univariate analysis were excluded. All studies with retrospective design, abstracts, case reports, conference presentations, editorials, reviews, and expert opinions were also excluded.

Various study and patient-related factors were extracted: number of participants; number of PPMI after TAVR; age; sex (male); number of patients with hypertension, diabetes mellitus, renal dysfunction or coronary artery disease; baseline procedural risk assessment by logistic EuroSCORE (European System for Cardiac Operative Risk Evaluation) or STS-PROM (Society of Thoracic Surgeons Predicted Risk of Mortality) score; number of patients with atrial fibrillation (AF), right bundle branch block (RBBB) or left bundle branch block (LBBB). We extracted the type of valve used - BEV [Edward Sapien (ESV), Sapien XT (XT) and Sapien 3 (S3)] versus SEV [Medtronic CoreValve Revalving System (MCRS) and Evolut R]. The data on valve size and various access sites used for TAVR (transfemoral, transapical, transaortic, trans-subclavian or trans-axillary) was also collected. Throughout the process we followed PRISMA (Preferred Reporting Items for Systematic Reviews and Meta-Analyses) guidelines for all stages of the design and implementation (supplement table 1). Research did not include human subjects and no Institutional Review Board approval was required.

\section{Assessed Predictors of PPMI}

The following factors were assessed as possible predictors: age; sex (male), hypertension, diabetes mellitus, baseline heart rate, prior aortic valve procedure, prior history of percutaneous coronary intervention (PCI), AF, RBBB, LBBB, left anterior fascicular block (LAFB), atrioventricular block (AVB), baseline interventricular conduction delay (IVCD), change in QRS duration, increased inter-ventricular septal dimension (IVSD), left ventricular outflow tract calcification for non-coronary, right and left coronary cusp, type of valve used SEV versus BEV, logistic EuroSCORE, post-procedural AVB, valve size/annulus size, depth of implant into the ventricle, and pre- and post TAVR balloon dilatation.

Certain factors assessed as predictors that were isolated from individual studies were not included: obesity, chronic obstructive pulmonary disease (COPD), mitral regurgitation, previous myocardial infarction, vascular disease, use of home oxygen, amiodarone, B-Blockers, presence of severe aortic stenosis, bicuspid and tricuspid aortic valve, pre-intervention bifascicular block, access route (transapical vs transfemoral vs transaortic), increase in balloon size and operative time.

\section{Statistical Analysis}

The Review Manager (RevMan) computer software [version 5.3]; Copenhagen: The Nordic Cochrane Centre, The Cochrane Collaboration, 2014; and R version 3.5.1 (R Development Core Team, 2010) was used to perform the meta-analysis. Odds ratios were extracted and converted into treatment effects and standard errors. The generic inverse variance method and a random-effects model was utilized. Results are presented as OR with $95 \%$ confidence intervals (CI). The consistency and heterogeneity were assessed by calculating $\mathrm{I}^{2}$, and publication bias was assessed by creating a funnel plot to evaluate for the existence of small-study effects. 


\section{RESULTS}

The initial search yielded 2,808 articles; after electronic and manual deduplication on title review 2,502 studies remained. An abstract review led to the exclusion of the least relevant articles, with 159 selected for full-text review. From that we identified 37 studies with 71,455 patients for which quantitative analysis was possible (figure 1) (6-42). All the studies were observational, and 23 out of 37 included patients who had undergone TAVR at a single institution (62\%). The incidence of PPMI after TAVR was $22 \%$. The mean age of our study population ranged between 76-84 years, with $52 \%$ female. Logistic EuroSCORE or the STS-PROM score was used to assess the pre-procedural risk in the majority of the studies. By contrast, in seven studies the authors reported no relevant information. Table 1 shows the baseline characteristics of patients in the included studies.

The type of valve implanted and the access routes used in each study are summarized in Table 2. In 17 studies SEV was used exclusively and BEV exclusively in 9; both valve types were used collectively in 11. Of the studies included in the analysis, the incidence of BEV was $64 \%$ compared to SEV 35.5\%, while other valves were $0.5 \%$. Fifty-four different predictors were eligible for the analysis, and two or more nonoverlapping datasets were available for 25 (supplement table 2). Results were divided based on demographic, electrocardiographic, imaging, valvular, and procedural predictors (figure 2).

\section{Demographic and Electrocardiographic Predictors}

The risk of PPMI after TAVR increased with age (OR 1.15, 95\% CI 1.01-1.32; $\mathrm{p}=0.04 ; \mathrm{I}^{2}=89 \%$ ), with history of diabetes mellitus (OR 1.08, 95\% CI 1.03-1.11; p < 0.01; $\mathrm{I}^{2}=0 \%$ ) and occurred with greater incidence in males compared to females (OR 1.29, 95\% CI 1.23-1.35; $\mathrm{p}=<0.01 ; \mathrm{I}^{2}=0 \%$ ). History of hypertension, COPD, past aortic valve procedure, or prior PCI and Logistic EuroSCORE were not found to be statistically significant predictors. In terms of ECG, presence of RBBB (OR 5.62, 95\% CI 3.9-8.10; p < $0.01 ; \mathrm{I}^{2}=82 \%$ ), baseline AVB (OR 1.75, 95\% CI 1.08-2.84; $\mathrm{p}=0.02 ; \mathrm{I}^{2}=91 \%$ ) and LAFB (OR 1.88, $95 \%$ CI 1.14-3.11; $\mathrm{p}=0.01 ; \mathrm{I}^{2}=7 \%$ ) were significantly associated with PPMI. No difference of risk was identified for patients with AF, LBBB, baseline IVCD or change in QRS duration following procedure.

\section{Imaging, Valvular and Procedural Predictors}

Presence of high calcium volume extending into LVOT in the area below the left coronary cusp (LVOT $\mathrm{lc})(\mathrm{OR}$ 4.21, 95\% CI 1.90-9.31; $\mathrm{p}<0.01 ; \mathrm{I}^{2}=0 \%$ ) and non-coronary cusp (LVOT $\mathrm{nc}$ ) (OR 3.15, 95\% CI 1.26-7.88; $\mathrm{p}=0.01 ; \mathrm{I}^{2}=89 \%$ ) was a significant predictor while presence of high calcium in the area below the right coronary cusp $\left(\mathrm{LVOT}_{\mathrm{rc}}\right)$ was not. Implantation of MCRS valve was associated with a 3.47 -fold increased risk of PPMI compared with ESV (OR 3.47, 95\% CI 1.75-6.88; p < 0.01; $\mathrm{I}^{2}=96 \%$ ). Implant depth (OR $1.24,95 \%$ CI 1.11-1.40; $\left.\mathrm{p}<0.01 ; \mathrm{I}^{2}=85 \%\right)$ and increased valve size/annulus size were also found to carry enhanced risk (OR 1.14, 95\% CI 1.02-1.27; $\mathrm{p}=0.02 ; \mathrm{I}^{2}=80 \%$ ); however, IVSD was not found to be a significant predictor. Pre-dilatation balloon valvuloplasty and post-implant balloon dilation were identified as notable predictors with OR $2.32\left(95 \%\right.$ CI 1.56-3.44; $\left.\mathrm{p}<0.01 ; \mathrm{I}^{2}=47 \%\right)$ and $1.76(95 \%$ CI $1.10-2.82 ; \mathrm{p}$ $\left.=0.02 ; \mathrm{I}^{2}=15 \%\right)$ respectively. Presence of post-procedure AVB was also found to be important (OR 3.08, $95 \%$ CI 1.34-7.10; $\left.\mathrm{p}<0.01 ; \mathrm{I}^{2}=90 \%\right)$.

\section{Discussion}

TAVR is rapidly emerging as a frequently performed and less invasive treatment alternative to surgical aortic valve replacement. In recent trials it has shown superior outcomes, even in patients with low surgical risk (43). However, despite the increase in procedural expertise, conduction disturbances and pacemaker insertion remain major complications following TAVR, with incidence reported to be approximately $7.8 \%$ to $20.3 \%$ in prior studies (44).

There have been several studies of post-TAVR PPMI looking for various risk predictors including anatomic, procedural, electrophysiological and demographic factors. A meta-analysis by Siontis et al (2014) included studies that identified 20 different predictors using both univariate and multivariable analysis (45). In our analysis, we included only those that used a multivariate regression model. Our findings suggest that age, 
baseline conduction problems (including RBBB, AVB and LAFB), LVOT calcium, pre- and post-procedure balloon dilatation, implant depth, valve size/annulus size and valve type are all linked to increased risk for PPMI following TAVR.

\section{Demographic and Electrocardiographic Predictors}

Age and sex were found to be significant in identifying patients at greater risk, as was diabetes. Interestingly other comorbidities such as hypertension, COPD, prior PCI, or prior aortic valve procedure were not.

Conduction defects are common during deployment of valves due to the close anatomic proximity of the aortic valve to the AV node as well as to the bundle of His. While many of these defects are transient, they can lead to heart block because the practice of keeping a temporary transvenous pacemaker post-procedure still exists. However, there is limited literature on how long a temporary pacemaker should be left in place before a decision is made for PPMI. Our report recognized the patients with underlying conduction defects of RBBB (identified as the most reliable predictor), LAFB, baseline, and post-procedure AVB as those with the greatest risk for needing PPMI. Particular focus should be given to underlying conduction defects while devising guidelines for the timing of pacemaker insertion.

\section{Imaging, Valvular and Procedural Predictors}

Heavy calcium volume has been identified as a predictor for pacemaker insertion in prior studies. However, different studies have used different parameters for the measurement of calcium burden including aortic valve calcium score, porcelain aorta, landing zone calcification, LVOT calcium, and mitral annulus calcification. In our analysis, high calcium volume in the area extending below $\mathrm{LVOT}_{\mathrm{lc}}$ and $\mathrm{LVOT}_{\mathrm{nc}}$ was found to be an important predictor. Our study also determined implant depth to be another major predictor suggesting the significance of higher deployment of the valve in the aortic area. Implant depth was reported in individual studies as either $>25 \%$ of stent frame below aortic annulus or $>6 \mathrm{~mm}$ length of an implant from the lower edge of the non-coronary cusp to the ventricular end of the prosthesis.

Increasing valve to aortic annulus oversizing ratios using multislice computed tomography (CT) is known to reduce the rate of paravalvular leak as the valve can fit better in the annulus (46). However, our analysis determined this size discrepancy, between the valve and the annulus, is associated with an increase in PPMI. The need for pre-dilatation balloon valvuloplasty has been substantially reduced as studies have shown direct TAVR is safe, feasible and has similar outcomes $(35,47)$. Our analysis shows that both pre and post balloon valvuloplasty are significant predictors of PPMI.

Since the introduction of TAVR, two valves have been widely used; the balloon-expandable ESV and the self-expandable MCRS. The rate of PPMI is markedly higher in the MCRS valve $(44,48)$. This higher rate has been attributed to the difference in stent design, long nitinol frame, and radial force exerted by the stent into the conduction tissue (49). The balloon-expandable technology has evolved from XT to S3, and the self-expandable technology has evolved to the Evolut $\mathrm{R}$ system. In the analysis of 12,381 patients from different trials and registries by Vlastra et al, new-generation BEV required PPMI less frequently when compared to the new-generation SEV (S3: $8.9 \%$ vs Evolut: 18.1\%). This difference was greater in earlygeneration valves (ESV: $6.1 \%$ and XT: $7.5 \%$ vs MCRS: $21.2 \%$ ) (44). The S3 valves were designed with a longer stent frame which lead to decrease in the paravalvular leak, but increased incidence of PPMI (7). Recently, modifications in the implantation technique by high deployment of the valve has led to a reduction in the rate of this complication (50).

\section{Study Limitations}

Certain limitations of our analysis should be considered. First, studies pooled in the report were all observational and susceptible to selection and confounding bias. Second, due to the wide selection of studies from all over the world with different expertise and devices, we expected clinical heterogeneity. However, we aimed to perform an umbrella review to identify all the predictors of PPMI post-TAVR that have remained significant in multivariable-adjusted models. Third, despite appropriate multivariable adjustment, residual confounding remains an issue from any observational data. We hope that our analysis adds enough statistical 
power to overcome the expected small study effects and confounders. Finally, the studies used in our analysis included both older and newer generation prostheses, so the risk factors identified could be generalized but not applied individually to each device.

\section{Conclusions}

Currently, there are no standard guidelines for the timing of PPMI following TAVR. The reviewed literature suggests a liberal use of PPMI immediately after TAVR, since a temporary pacemaker and more extended rhythm monitoring of all patients post-procedure can increase hospitalization days and costs and delay rehabilitation (49). Novel technology like Tempo pacemaker can be used safely for temporary pacing in ambulating patients for up to 7 days post TAVR, and needs further validation (51). Our meta-analysis identified 14 factors significantly associated with increased risk of PPMI following TAVR. This suggests that recognizing the high-risk population prior to performing TAVR, and targeting the risk factors that are modifiable, can help reduce the rate of PPMI.

\section{References}

1. Mack MJ, Leon MB, Thourani VH, et al. Transcatheter aortic-valve replacement with a balloonexpandable valve in low-risk patients. N Engl J Med. 2019;380(18):1695-1705.

2. Manoharan G, Van Mieghem NM, Windecker S, et al. 1-Year outcomes with the Evolut R selfexpanding transcatheter aortic valve: from the International FORWARD Study. JACC Cardiovasc Interv. 2018;11(22):2326-2334.

3. MacDonald I, Pasupati S. Transcatheter aortic valve implantation: know the differences between the currently available technologies. Eur Heart J. 2010;31(14):1663-1665.

4. van Gils L, Tchetche D, Lhermusier T, et al. Transcatheter heart valve selection and permanent pacemaker implantation in patients with pre-existent right bundle branch block. J Am Heart Assoc. 2017;6(3):e005028.

5. Tops LF, Schalij MJ, Bax JJ. The effects of right ventricular apical pacing on ventricular function and dyssynchrony implications for therapy. J Am Coll Cardiol. 2009;54(9):764-776.

6. Mauri V, Reimann A, Stern D, et al. Predictors of permanent pacemaker implantation after transcatheter aortic valve replacement with the SAPIEN 3. JACC Cardiovasc Interv. 2016;9(21):2200-2209.

7. Husser O, Pellegrini C, Kessler T, et al. Predictors of permanent pacemaker implantations and newonset conduction abnormalities with the SAPIEN 3 balloon-expandable transcatheter heart valve. JACC Cardiovasc Interv. 2016;9(3):244-254.

8. Fadahunsi OO, Olowoyeye A, Ukaigwe A, et al. Incidence, predictors, and outcomes of permanent pacemaker implantation following transcatheter aortic valve replacement: analysis from the U.S. Society of Thoracic Surgeons/American College of Cardiology TVT Registry. JACC Cardiovasc Interv. 2016;9(21):21892199 .

9. Nazif TM, Dizon JM, Hahn RT, et al. Predictors and clinical outcomes of permanent pacemaker implantation after transcatheter aortic valve replacement: the PARTNER (Placement of AoRtic TraNscathetER Valves) trial and registry. JACC Cardiovasc Interv. 2015;8(1 Pt A):60-69.

10. Mangieri A, Lanzillo G, Bertoldi L, et al. Predictors of advanced conduction disturbances requiring a late $([?] 48 \mathrm{H})$ permanent pacemaker following transcatheter aortic valve replacement. JACC Cardiovasc Interv. 2018;11(15):1519-1526.

11. De-Torres-Alba F, Kaleschke G, Vormbrock J, et al. Delayed pacemaker requirement after transcatheter aortic valve implantation with a new-generation balloon expandable valve: should we monitor longer?. Int $\mathrm{J}$ Cardiol. 2018;273:56-62.

12. Tichelbäcker T, Bergau L, Puls M, et al. Insights into permanent pacemaker implantation following TAVR in a real-world cohort. PLoS One. 2018;13(10):e0204503. Published 2018 Oct 17. 
13. Mauri V, Deuschl F, Frohn T, et al. Predictors of paravalvular regurgitation and permanent pacemaker implantation after TAVR with a next-generation self-expanding device. Clin Res Cardiol. 2018;107(8):688697.

14. Gensas CS, Caixeta A, Siqueira D, et al. Predictors of permanent pacemaker requirement after transcatheter aortic valve implantation: insights from a Brazilian registry. Int J Cardiol. 2014;175(2):248-252.

15. Gaede L, Kim WK, Liebetrau C, et al. Pacemaker implantation after TAVI: predictors of AV block persistence. Clin Res Cardiol. 2018;107(1):60-69.

16. Ramazzina C, Knecht S, Jeger R, et al. Pacemaker implantation and need for ventricular pacing during follow-up after transcatheter aortic valve implantation. Pacing Clin Electrophysiol. 2014;37(12):1592-1601.

17. Boerlage-Van Dijk K, Kooiman KM, Yong ZY, et al. Predictors and permanency of cardiac conduction disorders and necessity of pacing after transcatheter aortic valve implantation. Pacing Clin Electrophysiol. 2014;37(11):1520-1529.

18. Maan A, Refaat MM, Heist EK, et al. Incidence and predictors of pacemaker implantation in patients undergoing transcatheter aortic valve replacement. Pacing Clin Electrophysiol. 2015;38(7):878-886.

19. Kaneko H, Hoelschermann F, Seifert M, et al. Predictors of permanent pacemaker implantation after transcatheter aortic valve implantation for aortic stenosis using Medtronic new generation self-expanding CoreValve Evolut R. Heart Vessels. 2019;34(2):360-367.

20. Biviano AB, Nazif T, Dizon J, et al. Atrial fibrillation is associated with increased mortality in patients undergoing transcatheter aortic valve replacement: insights From the Placement of Aortic Transcatheter Valve (PARTNER) Trial. Circ Cardiovasc Interv. 2016;9(1):e002766.

21. Roten L, Wenaweser P, Delacrétaz E, et al. Incidence and predictors of atrioventricular conduction impairment after transcatheter aortic valve implantation. Am J Cardiol. 2010;106(10):1473-1480.

22. Guetta V, Goldenberg G, Segev A, et al. Predictors and course of high-degree atrioventricular block after transcatheter aortic valve implantation using the CoreValve Revalving System. Am J Cardiol. 2011;108(11):1600-1605.

23. Bleiziffer S, Ruge H, Hörer J, et al. Predictors for new-onset complete heart block after transcatheter aortic valve implantation. JACC Cardiovasc Interv. 2010;3(5):524-530.

24. D'Ancona G, Pasic M, Unbehaun A, Hetzer R. Permanent pacemaker implantation after transapical transcatheter aortic valve implantation. Interact Cardiovasc Thorac Surg. 2011;13(4):373-376.

25. Fraccaro C, Buja G, Tarantini G, et al. Incidence, predictors, and outcome of conduction disorders after transcatheter self-expandable aortic valve implantation. Am J Cardiol. 2011;107(5):747-754.

26. Khawaja MZ, Rajani R, Cook A, et al. Permanent pacemaker insertion after CoreValve transcatheter aortic valve implantation: incidence and contributing factors (the UK CoreValve Collaborative). Circulation. 2011;123(9):951-960.

27. Saia F, Lemos PA, Bordoni B, et al. Transcatheter aortic valve implantation with a self-expanding nitinol bioprosthesis: prediction of the need for permanent pacemaker using simple baseline and procedural characteristics. Catheter Cardiovasc Interv. 2012;79(5):712-719.

28. Mouillet G, Lellouche N, Lim P, et al. Patients without prolonged QRS after TAVI with CoreValve device do not experience high-degree atrio-ventricular block. Catheter Cardiovasc Interv. 2013;81(5):882-887.

29. Schroeter T, Linke A, Haensig M, et al. Predictors of permanent pacemaker implantation after Medtronic CoreValve bioprosthesis implantation. Europace. 2012;14(12):1759-1763.

30. Lange P, Greif M, Vogel A, et al. Reduction of pacemaker implantation rates after CoreValve@ implantation by moderate predilatation. EuroIntervention. 2014;9(10):1151-1157. 
31. Hayashida K, Morice MC, Chevalier B, et al. Sex-related differences in clinical presentation and outcome of transcatheter aortic valve implantation for severe aortic stenosis. J Am Coll Cardiol. 2012;59(6):566-571.

32. Akin I, Kische S, Paranskaya L, et al. Predictive factors for pacemaker requirement after transcatheter aortic valve implantation. BMC Cardiovasc Disord. 2012;12:87.

33. Bagur R, Rodés-Cabau J, Gurvitch R, et al. Need for permanent pacemaker as a complication of transcatheter aortic valve implantation and surgical aortic valve replacement in elderly patients with severe aortic stenosis and similar baseline electrocardiographic findings. JACC Cardiovasc Interv. 2012;5(5):540-551.

34. Calvi V, Conti S, Pruiti GP, et al. Incidence rate and predictors of permanent pacemaker implantation after transcatheter aortic valve implantation with self-expanding CoreValve prosthesis. J Interv Card Electrophysiol. 2012;34(2):189-195.

35. De Carlo M, Giannini C, Bedogni F, et al. Safety of a conservative strategy of permanent pacemaker implantation after transcatheter aortic CoreValve implantation. Am Heart J. 2012;163(3):492-499.

36. Muñoz-García AJ, Hernández-García JM, Jiménez-Navarro MF, et al. Factors predicting and having an impact on the need for a permanent pacemaker after CoreValve prosthesis implantation using the new Accutrak delivery catheter system. JACC Cardiovasc Interv. 2012;5(5):533-539.

37. Ledwoch J, Franke J, Gerckens U, et al. Incidence and predictors of permanent pacemaker implantation following transcatheter aortic valve implantation: analysis from the German transcatheter aortic valve interventions registry. Catheter Cardiovasc Interv. 2013;82(4):E569-E577.

38. Chorianopoulos E, Krumsdorf U, Pleger ST, Katus HA, Bekeredjian R. Incidence of late occurring bradyarrhythmias after TAVI with the self-expanding CoreValve(ß) aortic bioprosthesis. Clin Res Cardiol. 2012;101(5):349-355.

39. Pollari F, Großmann I, Vogt F, et al. Risk factors for atrioventricular block after transcatheter aortic valve implantation: a single-centre analysis including assessment of aortic calcifications and follow-up. Europace. 2019;21(5):787-795.

40. Kim WJ, Ko YG, Han S, et al. Predictors of permanent pacemaker insertion following transcatheter aortic valve replacement with the CoreValve Revalving system based on computed tomography analysis: an Asian multicenter registry study. J Invasive Cardiol. 2015;27(7):334-340.

41. Du F, Zhu Q, Jiang J, Chen H, Liu X, Wang J. Incidence and predictors of permanent pacemaker implantation in patients who underwent transcatheter aortic valve replacement: observation of a Chinese population. Cardiology. 2020;145(1):27-34.

42. Bisson A, Bodin A, Herbert J, et al. Pacemaker implantation after balloon- or self-expandable transcatheter aortic valve replacement in patients with aortic stenosis. J Am Heart Assoc. 2020;9(9):e015896.

43. Waksman R, Craig PE, Torguson R, et al. Transcatheter aortic valve replacement in low-risk patients with symptomatic severe bicuspid aortic valve stenosis. JACC Cardiovasc Interv. 2020;13(9):1019-1027.

44. Vlastra W, Chandrasekhar J, Muñoz-Garcia AJ, et al. Comparison of balloon-expandable vs. selfexpandable valves in patients undergoing transfemoral transcatheter aortic valve implantation: from the CENTER-collaboration. Eur Heart J. 2019;40(5):456-465.

45. Siontis GC, Jüni P, Pilgrim T, et al. Predictors of permanent pacemaker implantation in patients with severe aortic stenosis undergoing TAVR: a meta-analysis. J Am Coll Cardiol. 2014;64(2):129-140.

46. Abdel-Wahab M, Mehilli J, Frerker C, et al. Comparison of balloon-expandable vs self-expandable valves in patients undergoing transcatheter aortic valve replacement: the CHOICE randomized clinical trial. JAMA. 2014;311(15):1503-1514. 
47. Barbanti M. Early Outcomes of the Evolut R transcatheter aortic valve: a new technology between achieved goals and desirable improvements. JACC Cardiovasc Interv. 2017;10(3):283-285.

48. De Torres-Alba F, Kaleschke G, Diller GP, et al. Changes in the pacemaker rate after transition from Edwards SAPIEN XT to SAPIEN 3 transcatheter aortic valve implantation: the critical role of valve implantation height. JACC Cardiovasc Interv. 2016;9(8):805-813.

49. Athappan G, Patvardhan E, Tuzcu EM, et al. Incidence, predictors, and outcomes of aortic regurgitation after transcatheter aortic valve replacement: meta-analysis and systematic review of literature. J Am Coll Cardiol. 2013;61(15):1585-1595.

50. Bernardi FL, Ribeiro HB, Carvalho LA, et al. Direct transcatheter heart valve implantation versus implantation with balloon predilatation: insights from the Brazilian Transcatheter Aortic Valve Replacement Registry. Circ Cardiovasc Interv. 2016;9(8):e003605.

51. Webster M, Pasupati S, Lever N, Stiles M. Safety and feasibility of a novel active fixation temporary pacing lead. J Invasive Cardiol. 2018;30(5):163-167.

Figure Legends

Figure 1. PRISMA flowchart

Figure 2 . Forest plot for predictors of permanent pacemaker insertion after TAVR

\section{Hosted file}

2.Mahajan Tables.docx available at https://authorea.com/users/355434/articles/478665predictors-of-permanent-pacemaker-insertion-after-tavr-a-systematic-review-and-updatedmeta-analysis 


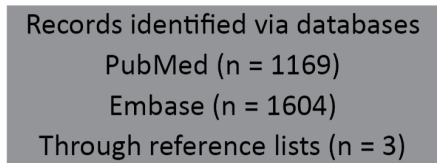

Through reference lists $(n=3)$
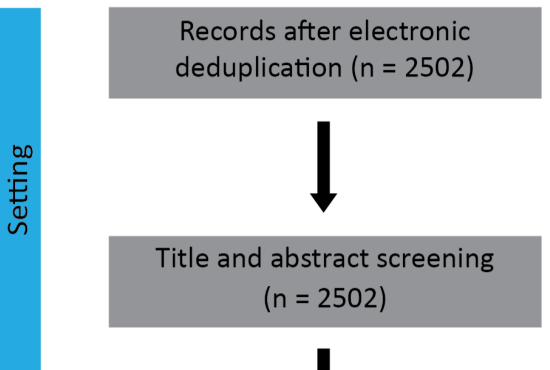

\section{Title and abstract screening} $(n=2502)$
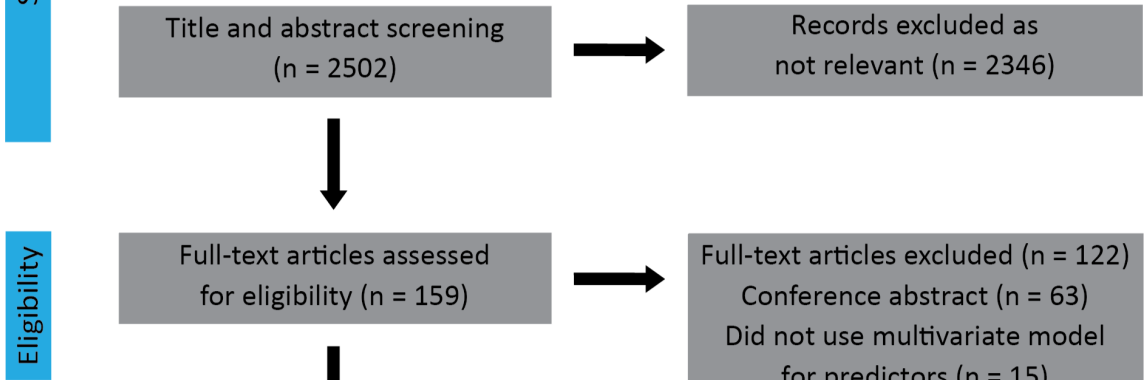

for eligibility $(n=159)$

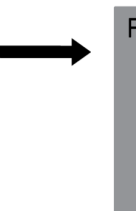

Full-text articles excluded ( $n=122$ )

Conference abstract $(n=63)$

Did not use multivariate model

for predictors $(n=15)$

Wrong outcome $(n=27)$

Review article $(n=4)$

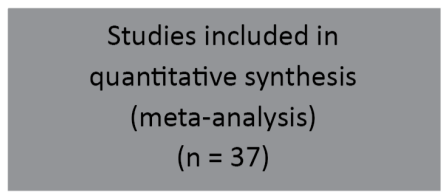

Duplicate data on manual deduplication $(n=13)$

Source

Age

Sex (Male)

Diabetes Mellitus

Hypertension

Chronic Obstructive Pulmonary Disease

Prior aortic valve procedure

Prior Percutaneous Coronary Intervention

Logistic Euro score

Right bundle branch block

Baseline AV conduction block

Post-procedure AV conduction block

Left anterior fasicular block

Atrial fibrillation

Left bundle branch block

Baseline interventricular conduction delay

Delta QRS

$1.00[0.84 ; 1.20]$

Left ventricular outflow tract calcification (left coronary cusp) 4.21 [1.90; 9.32$]$

Left ventricular outflow tract calcification (non coronary cusp) 3.15 [1.26; 7.88]

Left ventricular outflow tract calcification (right coronary cusp) $2.09[0.54 ; 8.10]$

Interventricular septal dimension

$0.78[0.35 ; 1.74]$

Self expandable valve vs Balloon expandable valve

$3.47[1.75 ; 6.88]$

Valve size/Annulus size

$1.14[1.02 ; 1.27]$

Pre-procedure balloon dilatation

Post-procedure balloon dilatation

$2.32[1.56 ; 3.45]$

$1.76[1.10 ; 2.82]$

Implantation depth

$1.24[1.10 ; 1.39]$

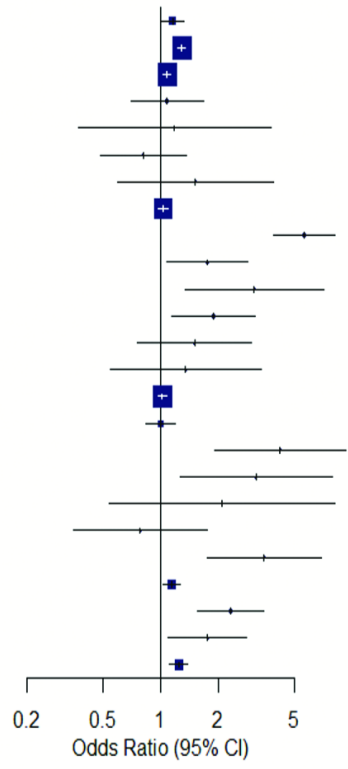

